\title{
Desarrollo rural para horticultores bonaerenses. El caso del programa "Cambio Rural INTA” en el partido' del Pilar (provincia de Buenos Aires, Argentina)
}

María Carolina Feito (CONICET/UBA)

Considerando las políticas sociales y las intervenciones de desarrollo como procesos continuos, negociados y socialmente construidos, que recogen iniciativas tanto "desde abajo" como "desde arriba", analizamos la constitución de intervenciones en la producción hortícola bonaerense, cuyos productores demandan acciones institucionales distintas a las de otras regiones del país, por su particular historia y estrategia socioproductiva. Intentamos identificar desde qué enfoque/s se concibe el desarrollo rural en la intervención y cómo opera esto en los cambios en la producción hortícola en el programa Cambio Rural de INTA, en el partido del Pilar. Analizamos posibilidades de sustentabilidad de esta intervención, en función del modelo de gestión implementado. Utilizamos metodología cualitativa con realización de trabajo de campo etnográfico en distintos ámbitos del partido². Considerando a los pequeños productores hortícolas como sujetos centrales de la intervención, la trayectoria y cobertura en términos de cantidad de población objetivo asistida es una fuente de legitimación de los discursos y visiones de los organismos estatales involucrados. La participación de los productores fortalece la toma de decisiones "desde abajo", en un modelo de desarrollo focalizado en la inclusión de los productores, sumando asistencia técnica y contención social.

\section{EL CONCEPTO DE DESARROLLO COMO UNA CONSTRUCCIÓN SOCIAL}

Durante la última década del siglo XX el concepto de desarrollo fue revisado y discutido desde diversas perspectivas que intentaron mostrar la relación entre el fracaso y los efectos perversos de tantas políticas y proyectos, así como la carga semántica, prejuicios culturales, sobreentendidos y simplificaciones de este 
dos connotaciones diferentes (Viola 2000): por un lado, el proceso histórico de transición hacia la economía capitalista; por otro, el aumento de la calidad de vida, la erradicación de la pobreza y la búsqueda de mejores indicadores de bienestar material (Ferguson 1990). Esta relación entre ambos fenómenos resulta insostenible si se considera la evidencia histórica y etnográfica que demuestra que el proceso de modernización aplicado durante la segunda mitad del siglo XX en los países del Tercer Mundo ha extendido la pobreza y la marginación social hasta límites sin precedentes. Lo destacable es que la fetichización del concepto de desarrollo actúa como un poderoso filtro intelectual de nuestra percepción del mundo contemporáneo (Viola 2000). Prejuicios tales como el economicismo (dada la centralidad de la teoría económica neoclásica en la configuración de las imágenes dominantes del desarrollo, especialmente identificando desarrollo y crecimiento económico) o el eurocentrismo se detectan en la mayoría de los diccionarios o documentos de trabajo de instituciones especializadas. De tal modo, cuando hablamos de desarrollo debemos considerar que este concepto presupone una determinada concepción de la historia de la humanidad, de las relaciones entre hombre y naturaleza, asumiendo al mismo tiempo un modelo implícito de sociedad considerado como universalmente válido y deseable (Viola 2000). Consideramos que "desarrollarse" implica expandir o realizar las potencialidades con que cuentan los sujetos y/o grupos sociales, accediendo gradualmente a mejoras cualitativas y potenciando identidades locales. Diferenciamos "desarrollo" de "crecimiento" en tanto este se refiere a un incremento cuantitativo a escala física.

\section{AGENTES Y ACTORES SOCIALES EN TÉRMINOS DE LA PROBLEMÁTICA DEL DESARROLLO}

La implementación de una intervención de desarrollo puede describirse como el momento en que diversos dominios sociales se entrecruzan y son mutuamente construidos. Este interfaz de diferentes actores permite ver arenas de conflicto, donde se negocian prácticas, discursos y sentidos, que a la vez modelan en cierta forma la intervención (Rodríguez Bilella 2004). En este contexto relacional es donde ejerce sus capacidades y desarrolla sus habilidades para influenciar a otros, regulando al mismo tiempo las influencias de los otros consigo mismo. Es decir, que realizará una estrategia y movilizará recursos dentro de un marco en el que todos los actores administran alguna cuota de poder, aunque sea mínima. En este enfoque, la relación de los actores con los agentes externos del desarrollo (agencias públicas, Ongs, iglesias, etc.) es un aspecto construido. En torno a este campo interactivo, opera un conjunto de factores. Uno de ellos es el concepto de agencia (Giddens 1984). Este se refiere a la fuerza o poder que provoca un resultado. La agencia desempeña un papel en el proceso de estructuración de la sociedad, produciendo y siendo producida por la estructura social. La noción de agencia no es sinónimo de capacidad de tomar decisiones, sino que además se requiere capacidad para organizar prácticas que permitan realizar las decisiones tomadas (Long 1992). La capacidad de agencia no está definida por la mera intencionalidad de hacer algo, sino por el poder de provocar el resultado. El concepto de agencia ha sido empleado para conciliar los conceptos de estructura y actor ${ }^{4}$. Long y van der Ploeg (1994) relacionan 
la administración de recursos que conduzcan al desarrollo de las mencionadas prácticas o el repertorio de estilos, formas culturales y racionalidades diferenciados y culturalmente construidos. Estas capacidades están permanentemente en conflicto/negociación con los modelos de intervención de las instituciones dedicadas al desarrollo. La posibilidad y el grado de superación de una capacidad de agencia externamente inducida dependen de la resolución de esta contradicción ${ }^{5}$. De ahí la importancia de estudiar el nivel micro de aplicación de los programas como lugar de intersección entre las áreas de incertidumbre naturalmente provocadas por los problemas y necesidades de la población y las áreas de incertidumbre artificiales o inducidas, consecuencia intencional de la intervención externa. Al analizar los vínculos establecidos por los destinatarios de un proceso de desarrollo a partir de una perspectiva del actor, surge la noción de práctica. Las prácticas deben interpretarse desde su capacidad de agencia.

\section{LOS PRODUCTORES HORTÍCOLAS DEL PARTIDO DE PILAR}

La provincia de Buenos Aires tiene el mayor cinturón hortícola del país, que se extiende en forma de anillo entre 30 y $100 \mathrm{~km}$ alrededor del área metropolitana, con significativa y creciente producción de cultivos bajo cubierta.

La cadena hortícola correspondiente al Territorio Periurbano Norte ${ }^{6}$ se concentra fundamentalmente en los alrededores de la ciudad de Buenos Aires, existiendo también cantidad importante de pequeños polos productivos ubicados alrededor de las principales localidades del área, conformando lo que podría denominarse un "archipiélago hortícola" (García et al. 2009). La actividad se caracteriza por un esquema de producción principalmente intensivo y altamente diversificado en cuanto a la forma de producción y a la cantidad de especies cultivadas. El destino de la producción es casi exclusivamente el consumo en fresco, dada la necesidad de abastecer diariamente una población de algo más de 13 millones de personas ubicadas en el área metropolitana. Parte de la misma se comercializa en mercados del interior de la provincia de Buenos Aires y en algunos casos más allá. Esta región ofrece hortalizas todo el año, con matices según la época, a fin de mantener oferta constante tanto en calidad como en diversidad de productos.

En relación a las características socioproductivas, las quintas hortícolas presentan diferencias según la zona y el tipo de establecimiento. Existe en general un perfil socioeconómico dominado por productores de mediana edad y bajo nivel educativo, de los cuales una alta proporción vive en sus establecimientos y, en la mayoría de los casos, no desempeña otra actividad económica, predominando las unidades productivas de pequeñas y medianas superficies, de entre 2 y 10 ha.

El partido o municipio del Pilar, creado en octubre de 1864, con $352 \mathrm{~km}^{2}$ de superficie, se encuentra al noroeste de la provincia de Buenos Aires, a $58 \mathrm{~km}$. de la Ciudad de Buenos Aires, en la tercera corona de la Región Metropolitana. Gobernado por el intendente Humberto Zuccaro, del Partido Justicialista, tenía en 2001232.463 habitantes, con una densidad de población de $654,83 \mathrm{hab} / \mathrm{km}^{2}$ (INDEC 2001). La zona es una de las de mayor crecimiento demográfico de la provincia en las últimas décadas: se calculaba a mediados de 2006 que el distrito 
ya alcanzaba unos 266.500 habitantes y se estima que en 2020 alcanzará el medio millón (Barsky \& Vio 2007). Por su inmejorable posición geográfica, la gran extensión y características de sus tierras y su ubicación estratégica sobre la Ruta Panamericana, atrajo en los últimos años a miles de personas que buscan tranquilidad y excelente acceso (principalmente desde la ciudad de Buenos Aires), como también a empresas e industrias para instalar sus plantas, especialmente en el Parque Industrial (Municipalidad del Pilar 2006). El partido se caracteriza también por su relativa cercanía y buena conectividad con la principal ciudad del país; la creciente heterogeneización social del medio rural; y la embrionaria intervención del municipio en el plano del Desarrollo Local. Este partido muestra simultáneamente el proceso de avance de la ciudad en forma de urbanizaciones campestres y otras ocupaciones con inversiones costosas, y la expansión de barrios pauperizados (Ringuelet, 2008). En su mayoría son explotaciones familiares con presencia de relaciones de mediería y aumento de la difusión del invernáculo. Se destinan 1.185 ha a la producción agropecuaria, de las cuales 467,9 (40\%) producen hortalizas frescas destinadas al mercado (un tercio de la superficie hortícola de la zona norte de la Región Metropolitana, lo cual marca su nivel de especialización) (Aboitiz 2009).

La horticultura en el partido es relativamente nueva y se desarrolla especialmente en las zonas de Parque Industrial Pilar, en Ruta 25 camino a Moreno y en Zelaya. Existen 576,3 ha dedicadas a la horticultura, 11,3 ha a la floricultura y 6,4 ha a viveros, de un total de 127 explotaciones hortiflorícolas, de las cuales 106 son hortícolas puras (Censo Hortiflorícola 2005 de la Pcia de Bs As). De un total de 761,7 ha (incluyendo superficie de vivienda y otros usos), 568,4 (75\%) están bajo régimen de arrendamiento. El aporte migratorio boliviano a esta actividad data de mediados de los ‘ 80 , como resultado de la atracción por las actividades económicas en Argentina (Benencia et al. 1997; Benencia y Gazzoti 1995; Feito 1999). Actualmente, de las 58.000 familias que habitan Pilar, se estima que entre 2.000 y 2.500 corresponden a núcleos bolivianos. Estos mayormente se encuentran al frente de explotaciones familiares cuya forma de tenencia corresponde en su mayoría a la figura del arriendo. En los últimos años, esta colectividad extendió su poder de decisión hacia el eslabón más importante de la cadena agroalimentaria hortícola: la comercialización (Feito, Díaz Galán y Diez Brodd 2009). Un porcentaje significativo de los canales de comercialización hortícolas de Buenos Aires se encuentra bajo influencia o control de estos migrantes (Barsky 2008; Pizarro 2007).

En este distrito, así como en el vecino Escobar, los migrantes bolivianos comenzaron a trabajar como peones y medieros en las quintas de productores portugueses en los años ‘60, y a partir de allí comienzan a desplazarlos gradualmente de la actividad hortícola. Una segunda oleada de inmigrantes arribó a principios de los ‘90 y es la que tiene actualmente presencia mayoritaria en las explotaciones. Estos experimentaron un ascenso económico, pasando sucesivamente de peones a patrones y de arrendatarios a propietarios, que Benencia denominó "escalera boliviana" (Benencia et al. 1997). Actualmente aprovechan el valor del arrendamiento que se ofrece en tierras periurbanas, espacios "en pausa" que están a la espera de ser valorizados por el capital, pero cuya concreción no necesariamente se realiza a corto plazo. Tanto el frente de avance de la urbanización desde la ciudad como el frente de avance de la soja desde el campo, un verdadero "juego de pinzas desde adelante y 
desde atrás" (Barsky et al. 2009), representan peligros concretos para el sostenimiento del cinturón verde. De ahí la importancia de sostener esta producción periurbana mediante una política municipal y metropolitana de suelos que considere estas complejidades. Se vislumbra un proceso de movilidad territorial de la colectividad boliviana, que se traslada progresivamente desde Escobar (primer centro de asociativismo iniciado a mediados de 1980) hacia Pilar y, paulatinamente, hacia la cuarta corona del conurbano, Exaltación de la Cruz ${ }^{7}$, acompañando los cambios en el mercado de tierras, el avance de las urbanizaciones para sectores de alto poder adquisitivo y un creciente poder de la comunidad de Pilar.

La colectividad boliviana conformó en Pilar la "Cooperativa de Provisión de Horticultores 2 de septiembre", creada en el año 2000, que tiene alrededor de 300 socios. Esta organización desarrolló un mercado concentrador ubicado estratégicamente sobre la ruta provincial 25, a 10 minutos del centro de Pilar, en un predio de 2 ha, adquirido en propiedad, en el que construyeron un galpón de material ${ }^{8}$. Unos 180 puestos generan ingresos para unas 800 personas, combinando horarios de funcionamiento con otros mercados bolivianos del conurbano ${ }^{9}$. El municipio articula actividades con los miembros de la Colectividad a través de un representante de la Secretaría de Ambiente y Calidad de Vida, y lo mismo ocurre con técnicos del programa Cambio Rural, ya que varios de los puesteros tienen también quintas y participan de los grupos del programa en el partido.

\section{LOS PROGRAMAS DE DESARROLLO RURAL EN EL PARTIDO DEL PILAR}

Los productores del cinturón hortícola bonaerense tienen una problemática particular y distintiva dentro de su propia provincia (Benencia et al. 1997) demandando acciones institucionales específicas, por su diferente realidad productiva y especialmente por su particular historia y estrategia socioproductiva (Feito1999) ${ }^{10}$. Los programas de desarrollo rural debieron readaptarse por las necesidades diferenciales del sector hortícola: producción altamente perecedera; proceso de trabajo de cortos períodos; presencia de medieros (Feito 2005).

Las permanentes transformaciones en el escenario del periurbano dificultan la adecuación de los pequeños productores hortícolas a dichos cambios. Diversas innovaciones tecnológicas generaron sobreoferta de productos con la consiguiente reducción de precios en un mercado poco previsible y poco transparente. A esto se suma la desvalorización social de la actividad y un mercado laboral precarizado, que conllevan la realización de las tareas en condiciones adversas. Actualmente, confluyen en la región Periurbano Norte acciones implementadas por distintas instituciones con la intención de mejorar las prácticas agrícolas en el sector: municipio, a través de las acciones de la Secretaría de Medioambiente y Calidad de Vida (Feito 2009); gobierno nacional, desde el Ministerio de Desarrollo Social de la Nación, con los programas Manos a la Obra"11 y Plan Jefes y Jefas de Hogar; INTA, mediante el programa Cambio Rural y PROAAS. Los objetivos diseñados apuntan a mejorar la capacitación y la organización de la producción en función de una mejora en los ingresos de los productores. 
El “Programa Federal de Reconversión Productiva para la Pequeña y Mediana Empresa Agropecuaria, Cambio Rural" (CR) fue creado en mayo de 1993 por la entonces SAGyP, delegando en el INTA los aspectos administrativos y técnicos de su ejecución y confiando a los Gobiernos Provinciales, entidades agropecuarias y al propio INTA su coordinación y supervisión. Su objetivo es “colaborar con los pequeños y medianos productores agropecuarios en la búsqueda de alternativas que permitan superar la crisis que los afecta e insertarse en un proceso de desarrollo sustentable en el contexto de una economía abierta. Se pretende que los productores generen mayores excedentes económicos para incrementar el nivel de vida de sus familias, ampliar las fuentes de empleo y retomar el proceso de inversión, asegurando el uso racional de la base agroecológica, en un cuadro de equidad social" (SAGyP/INTA/UC 1995). CR no constituye un sistema de subsidio; el Estado invierte a través del programa en procesos de asesoramiento, capacitación y organización de las estructuras productivas y comerciales, para mejorar la competitividad de las empresas y de la región, para lograr un mejor posicionamiento en los mercados internos y externos. Constituye una estrategia de intervención estatal selectiva, apuntando a aquellos grupos más vulnerables del sector agropecuario, en un contexto tendiente a fomentar la competitividad a nivel internacional y la eficiencia económica interna. También se lo puede considerar dentro de las denominadas políticas de inserción, que siguen una lógica de discriminación positiva porque se focalizan en poblaciones particulares y zonas singulares del espacio social, desplegando estrategias específicas (Castel 1997). Este programa desarrolla tareas en la zona hortícola desde mediados de los '90, trabajando con metodología grupal participativa (Feito 2005).

En el ámbito local, en junio 2006 el INTA firmó un convenio con la Municipalidad de Pilar para implementar el Programa "PROAAS" (Promoción de la Actividad Agropecuaria Sustentable) desde una visión integral donde confluyen la sostenibilidad económica, social y ambiental (Feito 2009). Este programa centra su objetivo principal en adecuar la actividad agropecuaria del distrito dentro de las denominadas Buenas Prácticas Agropecuarias $(B P A)^{12}$, confeccionando cada productor un cuaderno de campo provisto por el Municipio (Barsky et al. 2009). En una primera etapa de implementación se han conformado, con intervención del INTA, 9 grupos de horticultores que participan del programa $\mathrm{CR}$, ubicados en 8 áreas territoriales bien definidas, que reciben asistencia técnica gratuita por parte de 6 Promotores Asesores, que trabajan en Pilar desde abril de 2007, coordinados por un Agente de Proyecto. En materia de impacto ambiental la ordenanza 247/06 (marco legal del PROAAS) determina compatibilizar la actividad agropecuaria del distrito con el creciente desarrollo urbano, desde una visión de sostenibilidad ambiental de los procesos que impactan la región. También trabaja con cuestiones como la competitividad y la equidad social.

En abril de 2008 se firmó un Convenio Macro de Cooperación, Asistencia y Administración entre el INTA y la SAGPyA, y 8 municipios del Segundo Cordón Hortícola del Noroeste del AMBA (Mercedes, Luján, Moreno, General Rodríguez, Marcos Paz, Exaltación de La Cruz, Pilar y Escobar), con el cual la Nación responde a las necesidades del sector, promoviendo la articulación institucional a través de asistencia técnica y subsidios/créditos para los productores, encargándose del asesoramiento los técnicos del Programa CR del INTA. El convenio está destinado 
a fomentar y desarrollar la producción rural intensiva, especialmente hortiflorícola, como instrumento que combina la integración del Estado en los tres niveles, con la mirada puesta en los mercados, en una producción coordinada, con información e integración de los actores. El objetivo del convenio es producir alimentos accesibles para la producción y generar el desarrollo regional de los municipios, así como replicar la iniciativa en otras regiones del país. La iniciativa se asienta sobre tres ejes: el eje políticoinstitucional es novedoso y tiene el desafío de articular necesidades (de los productores, de los consumidores y de los gobiernos locales, provincial y nacional); el eje socioterritorial es el que tiene en cuenta el peso de la región periurbana y la necesidad de planificar el avance poblacional del Gran Buenos Aires; finalmente el eje de los planes de acción, donde aparecen el compromiso del Estado con el productor y el trabajo sobre aspectos que contribuyen a la sostenibilidad de la producción, como el ajuste del registro de productores ${ }^{13}$, la circulación de la información, la promoción del asociativismo, la formalización de la actividad y la aplicación de las BPA. Para el INTA, este convenio representa el desafío de integrarse con otros proyectos y otras instituciones, ante la falta de personal para abocarse a las nuevas tareas. Por ello, las herramientas que provee el programa CR son fundamentales para que los grupos de productores no se caigan una vez finalizada la financiación.

EI INTA trabaja en la región con la noción de "mesoterritorio" (como articulación de identidad, saberes locales, cultura, historia, instituciones, organizaciones), considerando el desarrollo como crecimiento rentable con equidad; las oportunidades como potencialidades de los productores y la competitividad como sistémica (asociar las empresas de un territorio para competir con las empresas de otros territorios). Se han detectado como problemas la falta de los siguientes elementos: canales alternativos de comercialización y mercados (en Pilar es difícil comercializar productos porque no hay fuerte demanda; a pesar de que hay productores, hay un solo mercado); normas legales aplicables al sector hortícola (no hay reglamentación para mediería); tecnificación, o sea, acceso del productor a maquinaria; zonificación para producción primaria (que debe subordinarse al mercado inmobiliario de los countries); ordenamiento territorial; estabilidad en precios; investigación y capacitación, para conocer la capacidad productiva de los recursos naturales de la región; mano de obra calificada (se desvalorizó el trabajo como insumo de la producción); acceso a crédito; terrenos para armar las huertas del Programa Prohuerta en zonas con elevada población. Otros problemas detectados son: existencia de políticas diferenciales en los distintos municipios de la región, lo que representa dificultades cuando hay productores de distintos municipios participando de un mismo grupo del programa; individualismo; informalidad — miedo a formalizarse por causa de los controles bromatológicos, sanitarios e impositivos-; deterioro de recursos naturales; problemas de tenencia (altos costos de arrendamiento, falta de contratos, inseguridad de continuidad en el predio). El INTA reconoce como oportunidades: potencialidad de articulación de actores de distintos territorios; trabajo asociativo de pequeños productores familiares (para buscar escala); necesidad de apoyo del Estado para potenciar la asociación; considerar la realidad de la demanda de productos; importancia alta de la horticultura en la región (por cantidad de familias, cantidad de empresas, valor de la producción, cantidad de hectáreas ocupadas, dinamización del trabajo); existencia de estructuras para reforzar la comunicación y la capacitación; posibilidad 
de generar productos exportables, de mejor calidad y con valor agregado; mejoramiento de condiciones de trabajo rural; promoción humana de familias de menores ingresos, soberanía alimentaria ${ }^{14}$.

EI INTA articula el trabajo en territorio con otras instituciones, que desempeñan roles de apoyo hacia los productores: distintas Universidades de la región (La Plata, Luján, Buenos Aires, Gral. Sarmiento), con las cuales CR tiene convenios para pasantías y asesoramientos; con el Ministerio de Desarrollo Social Nacional se gestiona apoyo financiero para el Mercado Boliviano de Pilar. El sector privado participa en la intervención en forma de organizaciones de productores (asociaciones civiles, cooperativas), y hay algunas ONGs trabajando en la zona, como el caso de SAHDES ${ }^{15}$. Existen algunas dificultades en la articulación: los organismos estatales están desbordados por demandas de la sociedad; existen problemas con el uso del agua y del suelo; falta de relevamiento sistemático de actores involucrados; falta de ordenamiento territorial; falta de infraestructura de salud, educación, transporte, etc.

Dentro del plan de trabajo integral de los grupos, se propuso la construcción de instalaciones móviles consistentes en depósitos de agroquímicos a nivel demostrativo, construidos con sistemas alternativos desarmables, con materiales desmontables, en función de la tenencia de la tierra como arrendatarios, lo cual permite su traslado si caduca el alquiler. Se permite mejorar la situación del productor y familia, desarrollando esquemas de higiene personal y posibilitando el mejoramiento de los productos manipulados, cumplimentando además un requisito legal incluido en la normativa de las BPA (Pineda 2009). En la práctica, los depósitos no fueron instalados en todas las quintas, provocando el recelo de aquellos que no fueron destinatarios de los materiales que entregaba el Municipio, y recayendo en los técnicos del INTA la supuesta responsabilidad por su falta. En aquellas en que se instalaron, no cumplieron su función original, dado que la mayoría de los productores continúa almacenando y utilizando agroquímicos de manera riesgosa (cerca o dentro de la vivienda, en lugares de tránsito de niños). Los técnicos consultados refieren que la medida es buena, pero habría que llevar a cabo una campaña de concientización del uso de los nuevos depósitos, así como mejorar los materiales y la forma de instalación. Los depósitos que vimos durante el trabajo de campo, recién instalados, tenían puerta sin cerradura, piso de material, paredes de aglomerado sin pintar y ventilación sin escurridor. "Las placas se doblaron, es difícil cerrar la puerta y pasar un candado, no tienen ventilación, no son seguros ni para guardar bicicletas" (productor de zona Zelaya).

Con el subsidio se armaron también tres Unidades Demostrativas de invernáculos, seleccionando a los productores destinatarios según su grado de compromiso con el programa y su predisposición a incorporar conocimientos que les permitan superarse, incluyendo el paquete de tecnologías que desde su quinta pueda incorporar adecuadamente. Ninguno de ellos tiene experiencia previa en el manejo de invernáculos. Como experiencia piloto, se implementa en tres grupos el Libro de Campo, el cual tiene por objeto documentar datos de cada explotación, incorporar fichas técnicas que comiencen a dar algunas pautas de Buenas Prácticas ${ }^{16}$.

Los subsidios en el partido fueron otorgados a quienes tenían habilitado el establecimiento hortícola, entregándose la misma partida a todos los municipios, a pesar de que Pilar es el de mayor cantidad de hectáreas 
implantadas y mayor cantidad de explotaciones. Además, en Luján y Gral. Rodríguez se entregó antes, lo cual generó disconformidad y reclamos por parte de los productores locales, que conocen la situación a través de parientes con quinta en otros partidos. Los técnicos solicitan ayuda a los investigadores para determinar elementos que permitan hacer una distribución más equitativa del subsidio.

Los reclamos de los productores se centran en los siguientes temas: falta de agua para riego; falta de herramientas ${ }^{17}$; problemas de comercialización - los productores llevan la mercancía todos al mismo tiempo, porque no hay planificación de las ventas, lo cual hace bajar los precios, debiendo rematar los productos al finalizar la jornada de venta:

“Tuvimos que volcar el repollo porque no camina, tiramos mucha verdura. Si son cajones de acelga, ellos (revendedores) en vez de llevar 5 llevan uno solo y lo ponen a \$1 el paquete, nosotros vendemos a \$3 el cajón. Ellos sacan $\$ 14$ y nosotros \$4. Lindo sería si en vez de comprarnos 1 nos compran 5, por no tirarlo, lo vendemos a cualquier precio. ¿Qué podemos hacer con esto? Queremos que no llueva más, porque cuando llueve, hay mucha falta de verdura y vendemos más. Sacamos 10 cajones pero valen más, los verduleros a veces empatan o pierden. A veces sacaba lo que compraba, a veces perdía. Muchos cerraron porque no les convenía" (productor de Pilar que comercializa en Mercado de Matanza).

Las distancias a los mercados les llevan mucho tiempo, lo cual a veces retrasa las labores en la quinta:

“Vendemos de 1 a 2 de la mañana, tenemos que salir para llegar temprano. Salgo 8 de la noche, porque en Matanza hay mucho delincuente; llego a las 10 de la noche al mercado a descargar hasta las 11 y media, guardamos el camión y a las 2 volvemos al puesto y a las 10 de la mañana cargamos de nuevo y llegamos a la quinta al mediodía, sin dormir. Eso 3 veces por semana, vamos cabeceando manejando en la ruta, nos agarra sueño al volver. Me agarró una vez la policía porque me dormí. De día, con los ruidos de la televisión y los chicos, no se puede dormir" (productor de Pilar que comercializa en Mercado de Matanza).

Las preocupaciones de los Agentes de Proyecto consisten en:

- revertir la informalidad de los productores - la precariedad de la contratación de mano de obra, de los arreglos de mediería, de los tipos de producción-;

- organizar a los productores;

- promover el registro de las actividades que se realizan en la explotación, mediante la concreción de un Diario de Campo: “Nosotros no anotamos nada, plantamos contra el tiempo, no planificamos. Si hay, gasto; si no hay, no compro más" (productor zona Zelaya);

- retener a los jóvenes en la quinta: “Mis tres hijos también trabajan, ellos nacieron arriba de las verduras, otra cosa no conocen. Parece que se van a cansar. Uno me dice: "por qué no vamos al barrio, que es más tranquilo, allá mis amigos juegan a la pelota, compramos un lotecito, una casita..." creo que con nosotros se termina la quinta" (productor de zona Zelaya); para ello, organizaron una capacitación para Jóvenes Emprendedores, conjuntamente con el Municipio;

- concientizar a los productores sobre la importancia de inscribirse en el RENSPA y de implementar 
en las quintas las BPA: “Si vienen sosteniendo la producción de esta forma hace 20 años, no van a adaptarse rápidamente, por lo tanto hay que respetar los tiempos del productor y adaptar las exigencias de las BPA a las características de la producción hortícola periurbana, generando manuales propios que disminuyan los estándares internacionales" (técnico a cargo de grupo CR).

Los técnicos pueden llevar muestras de agua o suelos para analizar en la Universidad de Luján; también informan sobre la posibilidad de compras conjuntas de semillas, abonos y fertilizantes, para bajar costos.

Mediante la intervención de los técnicos del INTA, los productores tienen posibilidades de realizar mejoras en la producción —implementación de BPA en sus quintas, mejores rendimientos, cuidado del medioambiente, posibilidad de sustentabilidad de la explotación_y en la comercialización de sus productos —nuevos canales informales, participación en otros mercados a los que antes no llevaban verdura-.

El cuello de botella para los extensionistas es definir el perfil de beneficiario de un programa como CR, concebido para Pymes. Se preguntan: ¿Qué es mejor? ¿Fortalecer y promover el liderazgo de los productores ya capitalizados? ¿Otorgar asesoramiento técnico a los no capitalizados o en vías de capitalización? ¿Atender a los que tienen algo de excedente y lo comercializan como pueden? ¿Ayudarlo a fortalecer su comercialización mediante un valor agregado (tecnología poscosecha, empaque, reconversión a la agroecología)?

Por su parte, el municipio rechaza las políticas asistencialistas y concibe a los productores como pequeños empresarios, predominando entre los funcionarios la idea de que dadas las características particulares de la producción hortícola - agricultura intensiva, productos perecederos, falta de tiempo disponible del productortienen que adaptar los programas: "Hay que hacer otro tipo de desarrollo para que los productores puedan solos, establecer niveles de articulación con otras instituciones, sino, no sirve" (funcionario municipal). Esta visión coincide con la de algunos técnicos del INTA, que pretenden transmitir a los productores que tienen en sus manos una pequeña empresa que deben aprender a gerenciar, con las condiciones impuestas por el SENASA (las BPA).

En términos generales, los productores valoran las condiciones de la intervención: “Gracias por todo el apoyo, por venir, por la visita"; "Gracias a Dios que vinieron ustedes, ahora me quedo más tranquilo para reclamar, ya sé que el problema fue la semilla que me vendieron" (productor que perdió toda una cosecha de tomate por comprar semilla en mal estado). La importancia de trabajar en conjunto es destacada por algunos para disminuir los costos de insumos. Sin embargo, algunos productores que entrevistamos y observamos en sus explotaciones no toman real conciencia de las ventajas de formar parte de un programa nacional. Se necesita replicar las capacitaciones informativas sobre los beneficios de ser miembro de un grupo de CR.

Se están produciendo importantes transformaciones en la estructura organizativa de la institución de desarrollo. En 2006, el Centro Regional Bs As Norte (CRBAN) del INTA, conjuntamente con Directores de Unidades, investigadores, extensionistas y el Consejo Regional, promueve el fortalecimiento de la extensión orientándolo al Desarrollo Rural con un enfoque territorial, de acuerdo a pautas de la Coordinación Nacional de Transferencia y Extensión. En 2007 el Consejo Regional aprueba la propuesta, actualmente en proceso de implementación, de la Unidad de Agricultura Urbana y Periurbana (AUPU) de la Región Metropolitana Bs As (AMBA). Se integra el 
Prohuerta AMBA al CRBAN, puesto que sus actividades se realizan fundamentalmente en su ámbito jurisdiccional. EI Consejo Directivo de INTA crea el AUPU AMBA a través de la Resolución 062/09, con el objetivo de "promover y acompañar la gestión estratégica del desarrollo con enfoque territorial, contribuyendo a la competitividad, al fortalecimiento de la soberanía y seguridad alimentaria, a la inclusión social y a la preservación y/o recuperación del medioambiente" (Martínez Quintana 2009). En función de la distribución radial de la población, el crecimiento demográfico y la identidad socioeconómica y cultural, se identifican cuatro territorios: Norte, Oeste, Urbano y Sur. Pilar forma parte del primero mencionado. En este sentido, creemos que atravesamos un momento histórico en el cual el INTA promueve una apertura hacia la agricultura periurbana como un tipo específico y particular, cuya problemática merece una intervención diferencial, así como la integración con equipos de trabajo de las Universidades, en un reconocimiento de la labor de investigación como herramienta de diagnóstico y planificación ${ }^{18}$.

\section{ANÁLISIS DE LA INTERVENCIÓN}

El caso relevado apoya la idea de la existencia de un proceso de crecimiento de la trama organizacional local, ya vislumbrado en trabajos anteriores (Nussbaumer 2008; Feito 2005; Díaz Galán, Diez Brodd y Feito 2009), conformado por la articulación entre organizaciones gremiales (asociaciones civiles) y organizaciones económicoproductivas (cooperativas), por un lado, y otras instituciones de la zona por el otro. Estos procesos contribuyen a revertir el histórico escaso protagonismo del Estado en la horticultura bonaerense y su responsabilidad en la dificultad para desarrollar alianzas económicas estratégicas como alternativa para enfrentar la crisis de los sistemas productivos. Se respeta la cultura y la experiencia previa de los productores, a la vez que se otorga importancia a la capacitación participativa, mediante la realización de talleres cuyos temas son priorizados por los mismos productores.

La definición de desarrollo local/territorial utilizada por INTA se refiere a un proceso de construcción social implementado por los actores del territorio que apunta a la generación de capacidades locales para aprovechar los recursos propios y movilizarlos hacia la satisfacción de las necesidades y los problemas de la comunidad. Este enfoque se basa en una concepción ampliada de lo rural, que integra tanto las actividades agropecuarias como las no agropecuarias. Tiene por objetivos principales: la creación de empleo y el aumento de la equidad social para mejorar la calidad de vida de los habitantes de una comunidad. Para ello, se trabaja en el fortalecimiento del capital social y del sistema económico productivo del territorio.

Como resultados posibles de las articulaciones, pueden mencionarse: trabajos abordados desde distintas disciplinas; obtención de información de otras instituciones útiles para el AUPU AMBA; mejor aprovechamiento de recursos; proyectos trabajados en forma conjunta interinstitucionalmente. En este sentido, la capacidad de los beneficiarios del desarrollo asociado a proyectos de crear y recrear normas de acción dentro de los 
programas evidencia su papel activo en el diseño y la administración efectivos de mecanismos regulatorios del comportamiento de todos los actores y su interacción, particularmente dentro del marco de proyectos específicos.

Se vislumbra la creación de un ámbito de discusión entre organizaciones públicas y la sociedad civil, concordando con cierta homogeneización del espacio en relación con el origen de los participantes —la mayoría del INTA, de la Secretaría de Medioambiente municipal y de organizaciones de productores-y concreción de actividades para llevar a cabo en forma conjunta, lo que permite la definición de una agenda programática recorridos regulares por las explotaciones por parte de los técnicos del programa; organización de talleres de capacitación; creación de canales comunes de comercialización de hortalizas-.

En el caso analizado, se destaca la "presencia en territorio" del INTA — definida por la trayectoria de sus técnicos reconocida por parte de los actores locales-. Considerando que el sujeto central de las acciones y programas de desarrollo rural local está constituido por los pequeños productores agropecuarios, la trayectoria y la cobertura en términos de cantidad de población objetivo asistida son una fuente de legitimación fundamental de los discursos y visiones de mundo de los organismos estatales vinculados (Nussbaumer 2008). La combinación de este reconocimiento con el "sentirse autorizado" para representar los intereses de los actores participantes muestra que cuanto mayor sea la "presencia territorial" en términos de cantidad de grupos y familias asistidas, mayor es la legitimidad de los discursos.

La articulación entre organismos públicos — distintas secretarías del gobierno municipal; el INTA- y asociaciones de la sociedad civil - cooperativa "2 de Septiembre", SAHDES, Universidades - como una nueva regla impone la confluencia en espacios comunes de discusión política, confrontando a cada uno de los agentes intervinientes a establecer estrategias para mantener sus posiciones en función de las reglas de juego en esta arena de disputa. En términos locales, la presencia en territorio y la trayectoria en políticas son recursos que contribuyen al reconocimiento de los agentes como conocedores y expertos en desarrollo rural. Estos recursos les permiten a su vez legitimarse como portavoces de las necesidades de los pequeños productores, y acumular capital en este sentido (Nussbaumer 2008). La participación de la sociedad civil, que fortalece un modelo de toma de decisiones de abajo hacia arriba, es bastante prometedora. Sin embargo, si bien existen convenios bilaterales, aún no se da una plataforma política común para el desarrollo rural. La articulación como nueva regla (Nussbaumer 2008), así como el nuevo rol que los representantes deben cumplir para investir la misma, todavía no es clara en el caso de Pilar, ya que no se define una dimensión operativa de las políticas de articulación en torno al desarrollo rural. Cada entidad pública participante confluye en espacios con otras organizaciones para la coordinación de políticas. Se comparte información, se acuerdan diagnósticos de estrategias de desarrollo, se asiste a diferentes reuniones y actos en forma conjunta, etc. Pero estas acciones no comprometen la autonomía en la toma de decisiones de las líneas y ámbitos de acción de sus organismos. Esto expresaría en abstracto la tradicional disociación entre la política social y la política económica, disociación que muestra dos mundos separados en la misma realidad rural: por un lado el del crecimiento económico; por el otro, aquel orientado a un modelo de desarrollo focalizado en la inclusión de los pequeños productores mediante la contención social. 


\section{REFLEXIONES FINALES}

La horticultura en Pilar está en una fase intermedia de desarrollo, registrando escasa innovación generacional y falta de mano de obra, en gran medida trasferida hacia el sector de la construcción. Esto amerita la necesidad de adaptar los programas de intervención a las particularidades de esta agricultura de proximidad (Barsky 2008). Si bien actualmente se está dando un momento histórico de apertura institucional del INTA hacia cuestiones territoriales, así como del municipio hacia el desarrollo local, existe un conjunto de desafíos tanto para el análisis de las condiciones sociopolíticas del municipio, como en el orden práctico, relacionados con la capacidad de acción concreta del gobierno local. Es necesario aumentar la capacidad técnica y de gestión mediante la formulación y la implementación de acciones que respondan a las demandas de la población más afectada. Se requiere ampliar los nexos con nuevas formas de organización social -ONGs, entidades intermedias, organizaciones de base, iglesias, universidades, etc.- y con el sector privado, desarrollando modelos participativos que articulen la relación Estado-sociedad en el plano local. Existen distintos desafíos, como intentar superar la divergencia de intereses estableciendo denominadores comunes mínimos para satisfacer al conjunto de los actores; o la construcción de una articulación público-privada que combine la promoción del desarrollo local con la inclusión de sectores desfavorecidos (Craviotti 2008).

Para el caso analizado, comprobamos que: i) la capacidad de los beneficiarios del desarrollo asociado a proyectos de crear y recrear normas de acción dentro de los programas evidencia su capacidad de agencia en el diseño y la administración efectivos de mecanismos regulatorios del comportamiento de todos los actores y su interacción, particularmente dentro del marco de proyectos específicos. Así, enriquecen o cuestionan los mecanismos estatales o se adaptan a ellos, de acuerdo a mecanismos regulatorios que ya conocen en sus prácticas grupales; ii) existen limitaciones institucionales referidas a la consideración que recibe el desarrollo rural en las políticas públicas, evidenciadas en la política de los '90, que resulta en la asignación de recursos para los pequeños y medianos productores en programas independientes y focalizados. Sólo una integración de estos programas permitiría superar estas limitaciones, en el marco de una mirada integral del sector.

Se destaca la implementación en el territorio de las intervenciones analizadas, mediante el modelo de gestión focalizado en la inclusión de los pequeños productores a través de la asistencia técnica y la contención social. Como alternativa a los modelos asistencialistas, en esta intervención se están desarrollando y generando capacidades productivas nuevas, así como potenciando las ya existentes, lo cual remite a las posibilidades de sostenibilidad de los resultados en el tiempo. Por otra parte, el enfoque con el que se concibe la intervención analizada considera el desarrollo como expansión y promoción de las potencialidades existentes. Esta concepción opera produciendo transformaciones en la producción hortícola del partido: mejorando los canales de comercialización existentes y creando otros nuevos; concertando la articulación interinstitucional; permitiendo el fortalecimiento institucional al interior del INTA y el fortalecimiento del asociativismo entre los productores, dado que la figura del técnico responsable es indispensable para obtener la habilitación municipal 
del establecimiento; aumentando la concientización entre los productores de la importancia de aplicar las Buenas Prácticas Agropecuarias, así como de las ventajas de participar de un grupo del programa.

Considerando las políticas sociales y las intervenciones de desarrollo como procesos continuos, negociados y socialmente construidos que incluyen iniciativas tanto "desde abajo" como "desde arriba" (Long 1992:35), la intervención analizada se constituye a partir de un complejo conjunto de relaciones, intereses e ideas socialmente definidas por los distintos actores implicados. Utilizando el análisis de interfaz, se comprende la forma en que los intereses, metas, percepciones y estrategias de diversos actores vinculados al programa resultan continuamente readaptados en función de las múltiples interacciones entre los actores locales y extralocales. El "modelo de grupo" sostenido por el programa en el caso estudiado intenta considerar las relaciones de poder existentes entre los productores, así como reconocer y articular las formas sociales existentes con su propia estrategia de intervención, incorporando las formas presentes de organización, comercialización y reproducción de los hogares. Sin embargo, las complejas interacciones entre los "proyectos" y prácticas de los actores, sus resultados esperados e imprevistos, crean tanto marcos habilitantes como limitantes de la acción social. Pretendemos que el diagnóstico realizado mediante un enfoque etnográfico permita que las intervenciones contribuyan a fortalecer la organización comunitaria, en pos de futuros reclamos a los poderes políticos.

Carolina Feito é doutora em Ciências Antropológicas pela 


\section{NOTAS}

1 A diferencia de las demás provincias de la Argentina, en la de Buenos Aires las divisiones territoriales se llaman partidos en lugar de departamentos. Estos también se constituyen en la división municipal de la misma. Los partidos-municipios cubren todo el territorio provincial, donde se utiliza el sistema de ejidos colindantes.

2 Entre septiembre-diciembre 2007 y junio-diciembre 2008 acompañamos a los técnicos del programa durante sus visitas semanales a los productores, las reuniones con los productores, las entrevistas a funcionarios municipales, etc.

3 Ver, por ejemplo: Cowen \& Shenton (1995), Escobar (1995, 1997) y Esteva (2000).

4 Esta discusión se da en el marco de la tradición sociológica y sus recientes revisiones que intentan explicar el vínculo entre el actor y el sistema social, llegando en algunos casos a negar la existencia misma de este último. Al respecto, ver un interesante resumen en Benencia \& Flood (2002).

5 El llamado "empoderamiento" (empowerment) es una capacidad de agencia externamente inducida, si bien suele reducirse como concepto a la concesión de oportunidades de participar en el acceso a bienes y servicios asimétricamente regulados (Benencia \& Flood 2002).

6 El denominado Territorio Periurbano Norte está integrado por los partidos de: Escobar, Pilar, Luján, Exaltación de la Cruz, Campana, Zárate y San Andrés de Giles, en toda su superficie o en parte de la misma, que equivale a un total de 5413 km2, con una población de 816.000 habitantes (INDEC 2006).

7 Actualmente la autora realiza una investigación sobre la implementación de los grupos de Cambio Rural para productores hortícolas en el partido de Exaltación de la Cruz.

8 Se gestionó un crédito ante el programa Manos a la Obra del Ministerio de Desarrollo Social nacional, con ayuda del Municipio y del Ministerio de Asuntos Agrarios de la provincia.

9 Funciona de lunes a sábados de 13:30 a 17:00 hs, con mayor movimiento los días lunes, miércoles y viernes, en los que la Comisión Directiva ha llegado a registrar, a través del personal de seguridad del predio, el ingreso de un promedio de 500 vehículos diarios, con picos de 750 vehículos.

10 Las políticas agrotecnológicas nacionales, que impulsaron la incorporación de maquinaria e insumos en función de aumentar la producción, influyeron también en el cinturón verde bonaerense, incrementando los rendimientos. Este aumento de la producción, asociado a su vez a una caída del consumo de verduras frescas registrada en las últimas dos décadas, produjo en el mercado una situación que se manifestó en concurrentes crisis de sobreproducción (Benencia \& Cattaneo 1989). Dichas crisis produjeron una importante caída de la rentabilidad, haciendo que muchos productores no pudieran obtener niveles de ingreso satisfactorios que les permitieran mantenerse en el mercado, especialmente en el estrato de los horticultores familiares medios. La desaparición de las explotaciones medias en el área bajo estudio se correspondió con un fuerte incremento de explotaciones más grandes o empresariales, lo cual estaría indicando la existencia de un proceso de diferenciación social en el sector.

11 El programa Manos a la Obra brinda apoyo económico financiero para la compra de insumos materiales y equipamiento, que se constituye como capital de trabajo para los pequeños emprendimientos productivos. Se canaliza a través de los subsidios para materiales, insumos y microcréditos, capacitación a los pequeños productores y asistencia técnica.

12 Las BPA son un conjunto de acciones destinadas a prevenir, reducir y controlar los peligros de contaminación biológica, química y/o física durante los procesos productivos. Incluidas en el Código Alimentario Argentino, forman parte de los Sistemas de Aseguramiento de la Calidad que contribuyen a garantizar productos inocuos. Los productores de hortalizas, frutas y aromáticas deben implementarlas gradual y obligatoriamente en un máximo de 5 años, establecidos por la Comisión Nacional de Alimentos (CONAL). En la producción primaria abarcan temas como: cultivo, semillas, agua segura, control de plagas, envases vacíos, instalaciones, herramientas y equipos, cosecha, vías de acceso, envases de productos, lavado, transporte, uso de agroquímicos o productos fitosanitarios, trazabilidad (rastreo), etc. En este marco, los técnicos de CR INTA brindan orientación general a los productores sobre la trazabilidad, documentación, cuadernos de campo y certificación de productos. 
13 La inscripción en el Registro Sanitario de Productores Agropecuarios (RENSPA) es requisito obligatorio para realizar actividades agropecuarias en Argentina. La inscripción se realiza en las oficinas del SENASA más cercanas a la explotación, presentando DNI y CUIT del productor, título de propiedad o contrato de locación o comodato que acredite su relación productiva con la ubicación del predio e identificación catastral del mismo. Según el cronograma gradual de inscripción, los productores de frutas, hortalizas y material de propagación, debieron comenzar a inscribirse en 2008.

14 Información recogida en el Taller INTA Escobar “Plan tecnológico regional 2009-2011. Territorio hortiflorícola diversificado", realizado en Escobar el 30 de septiembre de 2008, en el cual participó la autora.

15 SAHDER es una ONG que busca mejorar la calidad de vida de comunidades vulnerables, realizando acciones de educación y prevención en salud, mejora del hábitat (ambiente, barrio, vivienda), estudios sanitarios y fortalecimiento de la atención médica local pública, mediante la promoción comunitaria y articulación social, utilizando el arte y la educación como medios de transformación y realizando campañas sanitarias barriales, capacitando a vecinos socialmente activos como Promotores Comunitarios de Salud y coordinando su trabajo con instituciones locales (SAHDES). Su referente nos comentó el trabajo que realizan en la escuela pública de la localidad pilarense de Irizar. Recientemente tomaron contacto con los técnicos del programa CR de INTA para programar acciones comunes.

16 Se están preparando los Libros de Campo y las diferentes capacitaciones en materia de acopio y manipulación de agroquímicos y producción bajo cubierta.

17 Uno de los técnicos responsables de grupos nos comentó que la obtención de herramientas básicas como tractor, arado de reja y rastra les permitiría a los productores mejorar los tiempos de producción.

18 Por ejemplo, la creación del Foro de Universidades de la Agricultura Familiar Pampeana (del cual forma parte la autora de este trabajo) en el ámbito del IPAF Pampeana, y la convocatoria a investigadores universitarios a participar como parte del equipo de trabajo que confeccionó el Plan Territorio Periurbano Norte (en el cual también participó la autora, en un taller realizado en la Universidad Nacional de Luján- UNLU). 


\section{REFERENCIAS BIBLIOGRAFICAS}

ABOITIZ, P. 2009. "Caracterización de los productores hortícolas en el partido de Pilar". In Actas del IV Congreso Argentino y Latinoamericano de Antropología Rural. Mar del Plata.

BARSKY, A. 2008. “La bolivianización de la horticultura y los instrumentos de intervención territorial en el periurbano de Buenos Aires. Análisis de la experiencia de implementación de un programa de buenas prácticas agropecuarias en el partido de Pilar". In Actas del X Coloquio Internacional de Neocrítica. Universidad de Barcelona.

\& VIO, M. 2007. “La problemática del ordenamiento territorial en cinturones verdes periurbanos sometidos a procesos de valorización inmobiliaria. El caso del partido del Pilar, Región Metropolitana de Buenos Aires". In Actas del IX Coloquio Internacional de Neocrítica. Universidade Federal do Rio Grande do Sul.

A., ASTELARRA, S. \& GALVÁN, L. 2009. “Experiencias de intervención territorial en el cinturón hortícola de Buenos Aires. Análisis de la implementación del programa PRO.A.A.S. en el partido de Pilar". In Actas del IV Congreso Argentino y Latinoamericano de Antropología Rural. Mar del Plata.

BENENCIA et al. 1997. Área Hortícola Bonaerense. Cambios en la producción y su incidencia en los sectores sociales. Buenos Aires: Ed. La Colmena.

\& CATTANEO, C. 1989. La crisis de sobreproducción en el área hortícola bonaerense: causas, consecuencias y estrategias adoptadas para paliar sus efectos. Buenos Aires: Universidad de Buenos Aires, Facultad de Agronomía, Cátedra de Extensión y Sociología Rural.

\& GAZZOTTI, A. 1995. “Migración limítrofe y empleo. Precisiones e interrogantes”. Ponencia presentada en el V Congreso Argentino sobre Colectividades. Buenos Aires.

\& FLOOD, C. (comps.). 2002. Ongs y Estado. Experiencias de organización rural en Argentina. Buenos Aires: Ed. La Colmena.

CASTEL, R. 1997. La metamorfosis de la cuestión social: una crónica del salariado. Buenos Aires: Ed. Paidós.

COWEN, M. \& SHENTON, R. 1995. "The invention of Development" In J. Crush (comp.) Power of Development. Londres: Routledge.

CRAVIOTTI, C. 2008. “Participación, articulación, identidad. Desafíos emergentes para la incorporación de productores familiares en el contexto de estrategias de Desarrollo Local". In P. Rodríguez Bilella \& E. Tapella (orgs.) Transformaciones globales y territorios. Desarrollo Rural en Argentina. Experiencias y aprendizajes. Buenos Aires: Ed. La Colmena.

DÍAZ GALÁN, L., DIEZ BRODD, C. \& FEITO, M.C. 2009. “Organizaciones locales en el Área Hortícola Bonaerense: la acción colectiva como respuesta al conflicto social". In R. Benencia \& G. Quaranta (comps.) Producción, trabajo y territorio en el Cinturón Verde Bonaerense. Buenos Aires: Ed. Prometeo. 
ESCOBAR, A. 1995. Encountering Development, The Making and Unmaking of de Third World. Princeton: Princeton University Press.

1997. “Anthropology and Development". International Social Science Journal 154: 497-515.

ESTEVA, G. 2000. “Desarrollo”. In A. Viola (comp.) Antropología del desarrollo. Teorías y estudios etnográficos en América Latina. Barcelona: Ed Paidós Estudio.

FEITO, M. C. 1999. Juego de espejos. Un estudio antropológico sobre las estrategias de los sujetos sociales del sistema agroalimentario hortícola bonaerense. Tesis de Doctorado. Buenos Aires: Facultad de Filosofía y Letras, UBA.

2005. Antropología y desarrollo. Contribuciones del abordaje etnográfico a las políticas sociales rurales. El caso de la producción hortícola bonaerense. Buenos Aires: Ed. La Colmena.

2009. “Modalidades de intervención en el desarrollo local: productores hortícolas en el Municipio del Pilar, provincia de Buenos Aires". Ponencia presentada en el IV Congreso Argentino y Latinoamericano de Antropología Rural. Mar del Plata.

FERGUSON, J. 1990. The Anti-politics Machine. 'Development", Depoliticization and Bureaucratic Power in Lesotho. Cambridge: Cambridge University Press.

GARCÍA, M., LE GALL, J \& MIEREZ, L. 2009. “Reestructuración espacial y funcional en el AMBA: el archipiélago hortícola". Boletín Hortícola 14(41).

GIDDENS. A. 1984. The constitution of society: outline of the theory of structuration. Cambridge: Polity Press.

INDEC. 2001. Censo Nacional de Población y Vivienda.

LONG, N. 1992. "From paradigm lost to paradigm regained? The case for an actor-oriented sociology of development". In N. Long \& A. Long (orgs.) Battlefields of knowledge. The interlocking of theory and practice in social research and development. UK: Routledge.

\& VAN DER PLOEG, J.D. 1994. “Heterogeneity, actor and structure towards a reconstitution of the concept of structure". In D. Booth (ed.) New directions in social development. London: Longman.

MARTÍNEZ QUINTANA, O. 2009. “Crea el INTA una Unidad de Agricultura Urbana y Periurbana en la Región Metropolitana de Buenos Aires". Boletín Hortícola 14(41).

MUNICIPALIDAD DEL PILAR. 2006. Municipalidad del Pilar. Disponible en: <http://www.pilar.gov.ar/>.

NUSSBAUMER, B. 2008. "Estrategias de fortalecimiento institucional y políticas de desarrollo rural. El caso de Entre Ríos". In M. Manzanal, M. Arzeno \& B. Nussbaumer (Comps.) Territorios en Construccion: Actores, Tramas y Gobiernos entre la cooperacion y el conflicto. Buenos Aires: Ciccus.

PINEDA, C. 2009. "Gestión de la Calidad Agroalimentaria. Experiencias en la implementación de BPA". Boletín Hortícola 14(41). 
PIZARRO, C. 2007. “Inmigración y discriminación en el lugar de trabajo. El caso del mercado frutihortícola de la Colectividad Boliviana de Escobar". Estudios Migratorios Latinoamericanos 21(63): 211-243.

RINGUELET, R. 2008. "La complejidad de un campo social periurbano centrado en la zonas rurales de La Plata". Revista Mundo Agrario 9(17)

RODRíGUEZ BILELLA, P. 2004. “Etnografía y política social: el caso del enfoque orientado al actor". Ponencia presentada a las IV Jornadas de Etnografía y Métodos Cualitativos, IDES. Buenos Aires.

SADHES. Sahdes. Salud, hábitat, desarrollo. Organización civil. Disponível em: <http://www.sahdes.org/> .

SAGYP-INTA/UNIDAD DE COORDINACIÓN. 1995. Dos años de Cambio Rural: logros, limitantes y pautas para su fortalecimiento. Buenos Aires.

VIOLA RECASENS, A. (comp.). 2000. Antropología del desarrollo. Teorías y estudios etnográficos en América Latina. Barcelona: Editorial Paidós Studio. 
Desenvolvimento rural para horticultores bonaerenses. 0 caso do programa "Câmbio Rural INTA" em El Pilar (província de Buenos Aires, Argentina).

\section{RESUMO}

O presente trabalho analisa a constituição de intervenções na produção hortícola bonaerense. Tentamos identificar enfoques de desenvolvimento rural e a forma como estes influem nas mudanças de produção, no caso do programa Câmbio Rural do INTA no partido de El Pilar, cujos produtores demandam ações institucionais diferentes das implementadas em outras regiões do país, em função de suas particularidades históricas e das especificidades de suas estratégias produtivas. Analisamos, igualmente, as possibilidades de sustentabilidade dessa intervenção, em função do modelo de gestão implementado. Usamos uma metodologia qualitativa através da realização de trabalho de campo etnográfico. Considerando os horticultores como sujeitos centrais da intervenção, sua trajetória e a cobertura das ações em termos da quantidade de população atingida são uma fonte de legitimação dos discursos e das visões dos órgãos estatais envolvidos. A participação dos produtores fortalece a tomada de decisões "de baixo" num modelo de desenvolvimento focado na inclusão dos produtores, acrescentando-se assistência técnica e contenção social.

PALAVRAS-CHAVE: Antropologia rural; políticas governamentais de desenvolvimento; horticultura; Buenos Aires.

Development policies for Horticulturists: the Cambio Rural INTA program in El Pilar (Buenos Aires province, Argentina)

\section{ABSTRACT}

Based on ethnographic fieldwork, this paper analyzes governmental interventions in horticulture in Buenos Aires. We attempt to identify approaches to rural development and their impact in production practices, focusing on the program Cambio Rural do INTA (INTA's Rural Exchange Program) in the locality of El Pilar, where producers demand specific institutional actions, different from those implemented in other regions of the country, due to their historical particularities and specific production strategies. We also analyze the potential for sustainability of the management model that has been implemented, as well as the legitimacy discourses and visions of the state agencies involved. Regarding rural producers as central subjects of the intervention process, this model aims to strengthen bottom to top decision-making, combining technical knowledge and social commitment.

KEYWORDS: Rural Anthropology; Government policy development; Horticulture; Buenos Aires.

Recebido em 02/03/2010

Aprovado em 09/06/2011 